\title{
The CFTR M470V, Intron 8 Poly-T, and 8 TG-Repeats Detection in Chinese Males with Congenital Bilateral Absence of the Vas Deferens
}

\author{
Qiang Du, ${ }^{1}$ Zheng Li, ${ }^{2}$ Yongfeng Pan, ${ }^{3}$ Xiaoliang Liu, ${ }^{4}$ Bochen Pan, ${ }^{1}$ and Bin $\mathrm{Wu}^{5}$ \\ ${ }^{1}$ Department of Reproduction, ShengJing Hospital of China Medical University, Shenyang 110004, China \\ ${ }^{2}$ Department of Urology, RenJi Hospital, Shanghai Jiao Tong University School of Medicine, \\ Shanghai 200127, China \\ ${ }^{3}$ Shenyang Institute of Criminal Technology, Shenyang 110004, China \\ ${ }^{4}$ Department of Clinical Genetics, ShengJing Hospital of China Medical University, \\ Shenyang 110004, China \\ ${ }^{5}$ Department of Urology, ShengJing Hospital of China Medical University, Shenyang 110004, China
}

Correspondence should be addressed to Bin Wu; binwu_2013@126.com

Received 11 September 2013; Accepted 10 December 2013; Published 8 January 2014

Academic Editor: M. Ilyas Kamboh

Copyright (C) 2014 Qiang Du et al. This is an open access article distributed under the Creative Commons Attribution License, which permits unrestricted use, distribution, and reproduction in any medium, provided the original work is properly cited.

Purpose. To evaluate the significance of molecular detection of cystic fibrosis transmembrane conductance regulator (CFTR) M470V, intron 8 poly-T, and intron 8 TG-repeats in congenital bilateral absence of the vas deferens (CBAVD). Methods. Eighty-nine male patients with CBAVD and 103 healthy males were included in this study. Polymerase chain reaction was performed to amplify the polymorphic regions using primers from conserved regions. M470V was genotyped using real-time PCR by cycling probe. The exon 9 DNA sequence was determined using an automated sequencer. TG-repeats and poly-T were identified by direct sequencing analysis. Results. The 5T allele distribution was $0.32,0.66$ for $7 \mathrm{~T}$, and 0.02 for $9 \mathrm{~T}$ in CBAVD males, respectively. In contrast, the 5T allele distribution was $0.03,0.96$ for $7 \mathrm{~T}$, and 0.01 for $9 \mathrm{~T}$ in healthy control. Study of the polymorphisms of the upstream of exon 9 revealed a higher frequency of 5T allele in the CBAVD males. All cases with TG13T5 haplotype and TG12T5 homozygous led to CBAVD. The CFTR TG12T5-V470 variant haplotype was associated with CBAVD. Conclusion. The 5T allele of intron 8 of CFTR has clinically significant association with CBAVD. TG13T5 and TG12T5 homozygously led to CBAVD, and TG12T5-V470 may also lead to CBAVD.

\section{Introduction}

Congenital bilateral absence of the vas deferens (CBAVD; OMIM 277180) is found in $1 \%$ of infertile males and in up to $6 \%$ of those with obstructive azoospermia [1]. Extensive studies in western populations suggest that $75 \%$ of CBAVD cases are associated with mutations in the cystic fibrosis transmembrane conductance regulator (CFTR; OMIM 602421) gene, particularly the F508del, 5T, and R117H mutations [2]. However, prevalence of CFTR mutations among normal and infertile Chinese males is not well investigated. CBAVD may also be related to poly-T, TG-repeats, or M470V polymorphisms in the CFTR gene $[3,4]$. Shorter poly-T tracts in CFTR nontranslated regions are associated with reduced levels of normal CFTR mRNA and protein [5]. Individuals harboring the T5 allele exhibit aberrant mRNA splicing of CFTR exon 9, generating a subset of transcripts that lack this exon and specify a nonfunctional CFTR protein $[6,7]$. The incidence of the $5 \mathrm{~T}$ allele in the healthy Chinese population is known to be 0.0380 [8], but the distribution of the poly-T, TG-repeat, and M470V polymorphisms among Chinese CBAVD patients has not been described. Moreover, it remains unclear whether M470V and (TG) $\mathrm{mTn}$ are risk factors for CBAVD in Chinese males. In this study, the distribution of (TG)mTn among normal Chinese males and Chinese CBAVD patients was assessed using DNA sequencing to explore the relationships 
between CBAVD and the TG-repeat, poly-T, and M470V polymorphisms in the CFTR gene.

\section{Materials and Methods}

2.1. Sample Collection. This study was approved by the Medical Ethics Committee of Shengjing Hospital of China Medical University (Shenyang, China). All patients gave written informed consent. Eighty-nine male patients with unambiguous clinical diagnoses of CBAVD and 103 normal male participants who underwent assisted reproductive treatment owing to female infertility were enrolled in the study. They were treated at the Center for Assisted Reproduction of Shengjing Hospital (Shenyang, China) from Aug. 2008 to Jan. 2012. Screening criteria for CBAVD patients were as follows: (1) no signs or symptoms of cystic fibrosis (CF) other than primary infertility; (2) normal bilateral testicular size with bilateral absence of the vas deferens; (3) absence of detectable sperm as determined by 3 routine semen exams using centrifugal sedimentation microscopy; absence of sperm in the urinary sediment following ejaculation (thus retrograde ejaculation has been excluded), acidic semen $\mathrm{pH}$, and significantly lower quantitative of seminal fructose and glycosidase than normal; (4) normal levels of reproductive hormones and karyogram; and (5) recovery of sperm by aspirating from the epididymis or testis. Screening criteria for normal fertile male participants were as follows: (1) underwent assisted reproductive treatment due to female infertility; (2) normally developed bilateral testis; (3) normal routine semen test results; (4) normal karyogram.

2.2. Genomic DNA Extraction. Peripheral blood samples $(2 \mathrm{~mL})$ were collected from each subject and were anticoagulated with ethylenediaminetetraaceticacid (EDTA). Genomic DNA was extracted using the automated BioRobot EZ1 Workstation (Qiagen, Hilden, Germany) with the EZ1 DNA blood $200 \mu \mathrm{L}$ kit (Qiagen, Hilden, Germany). DNA concentrations were measured using UV spectrophotometry. DNA purity was validated by measuring the $A_{260} / A_{280}$ ratio, and samples measuring between 1.8 and 2.0 were retained. Samples failing this test were reextracted. DNA samples then were identified with serial numbers and stored at $-80^{\circ} \mathrm{C}$ until subsequent analyses.

2.3. Detection of M470V in the CFTR Gene with Real-Time $P C R$. The following primers flanking the M470V region of the CFTR gene were designed with Primer 5.0 software: sense $5^{\prime}$-CTTCTGCTTAGGATGATAATTGG- $3^{\prime}$ and antisense $5^{\prime}$-GCTTTGATGACGCTTCTGTA- $3^{\prime}$. Primers and corresponding fluorescent probes were synthesized by TaKaRa Biotechnology (Dalian, China). The probes were $5^{\prime}$-(FAM) cttctaatg (A) tg (Eclipse)-3' and $5^{\prime}$-(HEX) tctaatg (G) tga (Eclipse)-3'. The cycling probe method [9] was used to genotype samples for the M470V polymorphism. PCR products were analyzed by $3 \%$ agarose gel electrophoresis to confirm that specific bands had been amplified by the synthesized primers and probes. Real-time PCR products were sequence-verified to ensure that the primers and probes were effective in the detection of M470V. PCR was performed using a reaction solution specified by the CycleavePCR core kit instructions (TaKaRa Biotechnology). Components and conditions of the real-time PCR are briefly described and were the following: $1 \mu \mathrm{L}$ of the DNA sample, $2.5 \mu \mathrm{L} 10 \times$ Cycleave PCR Buffer, $3 \mu \mathrm{L}$ dNTP mixture $(2.5 \mathrm{mM}$ each), $5 \mu \mathrm{L} \mathrm{Mg}$ solution $(25 \mathrm{mM}), 0.5 \mu \mathrm{L}$ each primer pair $(10 \mathrm{uM})$, $1 \mu \mathrm{L}$ each probe pair $(3 \mathrm{uM}), 0.5 \mu \mathrm{L}$ Tli Rnase H II $(200 \mathrm{U} / \mu \mathrm{L})$, $0.25 \mu \mathrm{L}$ TaKaRa Ex Taq HS $(5 \mathrm{U} / \mu \mathrm{L})$, and $9.75 \mu \mathrm{L}$ RNase free $\mathrm{dH} 2 \mathrm{O}$. After initial denaturation at $95^{\circ} \mathrm{C}$ for $30 \mathrm{sec}$, the PCR system was given 50 cycles of denaturation at $95^{\circ} \mathrm{C}$ for $5 \mathrm{sec}$, annealing at $57^{\circ} \mathrm{C}$ for $15 \mathrm{sec}$, and extension at $72^{\circ} \mathrm{C}$ for $34 \mathrm{sec}$ and $5 \mathrm{~min}$. Samples were analyzed using an ABI 7500 Sequence Detection System (Applied Biosystems) in combination with a postreaction melting curve analysis.

2.4. Sequence Detection of Poly-T and TG-Repeats in the CFTR Gene. The following primers flanking the poly-T and TGrepeat regions of the CFTR gene were designed with Primer 5.0 software: sense $5^{\prime}$-TGGGCAAATATCTTAGTTT- ${ }^{\prime}$ and antisense $\quad 5^{\prime}$-GAGTACCAAGAAGTGAGAA- $3^{\prime}$. Primers were synthesized and sequenced by TaKaRa Biotechnology. The reaction components and conditions were the following: $1 \mu \mathrm{L}$ of the DNA sample, $25 \mu \mathrm{L} 10 \times$ Ex Buffer, $0.5 \mu \mathrm{L}$ each primer pair ( $3 \mathrm{uM}), 2.5 \mu \mathrm{L} \mathrm{dNTP} \mathrm{(2.5} \mathrm{Mm} \mathrm{each),} \mathrm{and} 20.5 \mu \mathrm{L}$ $\mathrm{dH} 2 \mathrm{O}$. The initial denaturation at $94^{\circ} \mathrm{C}$ for $60 \mathrm{sec}$ was followed by 40 cycles of denaturation at $94^{\circ} \mathrm{C}$ for $30 \mathrm{sec}$, annealing at $55^{\circ} \mathrm{C}$ for $30 \mathrm{sec}$, and extension at $72^{\circ} \mathrm{C}$ for $30 \mathrm{sec}$ and $120 \mathrm{sec}$. PCR products $(5 \mu \mathrm{L})$ migrated through an agarose gel as a single band of approximately $318 \mathrm{bp}$. Amplicons were purified using shrimp alkaline phosphatase and exonuclease I (TaKaRa Biotechnology) and subsequently were sequenced. Sequencing results were analyzed using Chromas v.1.62 software, and samples were individually confirmed manually. Paired-end sequencing was performed to verify sequences that could not be confirmed by the above approach.

2.5. Statistics. Data were evaluated with Pearson's chisquared test and Fisher's exact test using SPSS v.13.0 software. Significance was assessed at $P<0.05$.

\section{Results}

The clinical characteristics and genotypes of the patients in this study are shown in Table 1 . The MV genotype was the most prevalent, with a frequency of $44.66 \%$ among normal Chinese males, followed by the VV genotype, with a frequency of $35.92 \%$. The MM genotype was the least common, with a frequency of $19.42 \%$. The distribution of MM, MV, and VV genotypes among normal males was consistent with the Hardy-Weinberg equilibrium $(P=0.4065)$. The V470 allele occurred most often among normal male participants, with a frequency of $58.25 \%$. The distribution ratio of M470 and V470 alleles among normal males was approximately $0.7: 1$. We detected no statistically significant differences in the M470V genotype distributions or allele frequencies between 
TABLE 1: CFTR genotypes in Chinese CBAVD patients and males with normal fertility.

\begin{tabular}{|c|c|c|c|c|}
\hline M470V genotypes & Poly-T genotypes & $\left(\mathrm{TG}_{m}\right) \mathrm{T}_{n}$ genotypes & CBAVD & Normal fertility males \\
\hline $\mathrm{M} / \mathrm{M}$ & T5/T5 & TG13T5/TG13T5 & 1 & - \\
\hline $\mathrm{M} / \mathrm{M}$ & $\mathrm{T} 5 / \mathrm{T} 7$ & TG13T5/TG12T7 & 6 & - \\
\hline $\mathrm{M} / \mathrm{M}$ & T5/T9 & TG13T5/TG11T9 & 1 & - \\
\hline $\mathrm{M} / \mathrm{M}$ & T5/T9 & TG12T5/TG11T9 & 1 & - \\
\hline $\mathrm{M} / \mathrm{M}$ & $\mathrm{T} 7 / \mathrm{T} 7$ & TG11T7/TG12T7 & 1 & - \\
\hline $\mathrm{M} / \mathrm{M}$ & $\mathrm{T} 7 / \mathrm{T} 7$ & TG11T7/TG11T7 & 1 & - \\
\hline $\mathrm{M} / \mathrm{M}$ & $\mathrm{T} 7 / \mathrm{T} 7$ & TG12T7/TG12T7 & 10 & 19 \\
\hline $\mathrm{M} / \mathrm{M}$ & $\mathrm{T} 7 / \mathrm{T} 7$ & TG12T7/TG13T7 & - & 1 \\
\hline $\mathrm{M} / \mathrm{V}$ & $\mathrm{T} 5 / \mathrm{T} 5$ & TG13T5/TG12T5 & 7 & - \\
\hline $\mathrm{M} / \mathrm{V}$ & T5/T5 & TG12T5/TG12T5 & 1 & - \\
\hline $\mathrm{M} / \mathrm{V}$ & $\mathrm{T} 5 / \mathrm{T} 7$ & TG12T5/TG12T7 & 13 & 4 \\
\hline $\mathrm{M} / \mathrm{V}$ & $\mathrm{T} 5 / \mathrm{T} 7$ & TG12T5/TG11T7 & 2 & - \\
\hline $\mathrm{M} / \mathrm{V}$ & T5/T9 & TG12T5/TG10T9 & 1 & - \\
\hline $\mathrm{M} / \mathrm{V}$ & $\mathrm{T} 7 / \mathrm{T} 7$ & TG11T7/TG12T7 & 15 & 32 \\
\hline $\mathrm{M} / \mathrm{V}$ & $\mathrm{T} 7 / \mathrm{T} 7$ & TG11T7/TG13T7 & - & 1 \\
\hline $\mathrm{M} / \mathrm{V}$ & $\mathrm{T} 7 / \mathrm{T} 7$ & TG12T7/TG12T7 & 6 & 9 \\
\hline $\mathrm{V} / \mathrm{V}$ & T5/T5 & TG12T5/TG12T5 & 4 & - \\
\hline $\mathrm{V} / \mathrm{V}$ & $\mathrm{T} 5 / \mathrm{T} 7$ & TG12T5/TG12T7 & 1 & 2 \\
\hline $\mathrm{V} / \mathrm{V}$ & $\mathrm{T} 5 / \mathrm{T} 7$ & TG12T5/TG11T7 & 6 & 1 \\
\hline $\mathrm{V} / \mathrm{V}$ & $\mathrm{T} 7 / \mathrm{T} 7$ & TG11T7/TG12T7 & 6 & 10 \\
\hline $\mathrm{V} / \mathrm{V}$ & $\mathrm{T} 7 / \mathrm{T} 7$ & TG11T7/TG11T7 & 5 & 22 \\
\hline $\mathrm{V} / \mathrm{V}$ & T7/T7 & TG12T7/TG12T7 & - & 1 \\
\hline $\mathrm{V} / \mathrm{V}$ & T7/T9 & TG11T7/TG11T9 & 1 & - \\
\hline $\mathrm{V} / \mathrm{V}$ & T7/T9 & TG11T9/TG12T7 & - & 1 \\
\hline
\end{tabular}

Chinese CBAVD patients and fertile male participants $(P=$ 0.3177 and $P=0.1615$, resp.) (Table 2).

With regard to poly-T polymorphisms, T7 was the most common allele among normal Chinese male participants, with a frequency of $96.12 \%$ (Table 3). Of the TG-repeats, TG12 was most frequently represented among normal Chinese males (frequency $55.83 \%$ ), followed by TG11 (43.20\%). The allele frequency distributions of the T5 and TG13 alleles significantly differed between the two groups $(P<0.001$; see Table 3). T7/T7 was the most commonly detected haploid genotype among normal Chinese males (92.23\%); all T5/T5 were CBAVD (Table 4). TG12T7 was the most commonly detected haploid genotype among normal Chinese males (52.43\%), followed by TG11T7 (42.72\%) (Table 5). The frequency distributions of the TG13T5 and TG12T5 haploid genotypes differed significantly $(P<0.001)$. Among 89 CBAVD patients, 14 individuals were heterozygous for the TG13T5 haploid genotype and 1 individual was a TG13T5 homozygote. None of the 103 normal male participants harbored the TG13T5 haplotype. All 5 TG12T5 homozygotes identified were CBAVD patients.

In addition, the frequency distributions of the TG12T5V470 haploid genotypes differed significantly $(P<0.001)$ (only the homozygote of (TG)mTn and Tn could be analyzed in the genotype frequencies of haploid) (Table 6). Our results suggest that individuals with a TG12T5-V470 haploid genotype have a significantly higher risk of CBAVD $(P<0.001)$.
In the entire study population, none of the individuals carried the TG13T5-V470 haploid genotype particularly.

\section{Discussion}

The CFTR gene was originally cloned and identified by Riordan et al. in 1989 [10]. CFTR is located on chromosome $7 \mathrm{q} 31.2$ and is $188,704 \mathrm{bp}$ in length [11], spanning 26 introns and 27 exons. The CFTR gene encodes a 6,132-bp mRNA that specifies the 1,480-residue CFTR protein. The CFTR protein has a relative molecular weight of approximately 168,173 . The in vivo function of the CFTR protein is determined by its genotype [12].

CBAVD, a CF-related disorder (OMIM 219700) [13], is an important congenital cause of male infertility and obstructive azoospermia, which correlated closely with specific CFTR mutations in western populations, mainly R117H, delF508, and 5T, which are distributed differently in different subgroups of people [2]. CFTR gene analyses have focused primarily on the intron 8 polymorphism, poly-T (IVS8 polyT), as well as TG-repeats, and the M470V allele. However, only 4 studies have been performed to investigate the poly$\mathrm{T}$ polymorphism and congenital bilateral absence of the vas deferens in Chinese patients [14-17].

Interestingly, although the poly-T polymorphisms in those studies were similar, the frequency data differed greatly from each other and this could be because those studies were 
TABLE 2: Genotypes and allele frequencies at M470V polymorphic site.

\begin{tabular}{|c|c|c|c|}
\hline & $\begin{array}{c}\text { CBAVD (frequencies) } \\
(2 n=178)\end{array}$ & $\begin{array}{c}\text { Control (frequencies) } \\
(2 n=206)\end{array}$ & $P$ \\
\hline \multicolumn{4}{|c|}{ Frequencies of individuals with genotypes } \\
\hline MM & $21 / 89(23.60 \%)$ & $20 / 103(19.42 \%)$ & \multirow{3}{*}{0.3177} \\
\hline $\mathrm{VV}$ & $23 / 89(25.84 \%)$ & $37 / 103(35.92 \%)$ & \\
\hline MV & $45 / 89(50.56 \%)$ & $46 / 103(44.66 \%)$ & \\
\hline \multicolumn{4}{|c|}{ Allele frequencies at M470V polymorphic site } \\
\hline M & $87 / 178(48.88 \%)$ & $86 / 206(41.75 \%)$ & \multirow{2}{*}{0.1615} \\
\hline $\mathrm{V}$ & $91 / 178(51.12 \%)$ & $120 / 206(58.25 \%)$ & \\
\hline
\end{tabular}

TABLE 3: Allele frequency of poly-T and TG-repeats polymorphisms.

\begin{tabular}{lccc}
\hline & $\begin{array}{c}\text { CBAVD (frequencies) } \\
(2 n=178)\end{array}$ & $\begin{array}{c}\text { Control (frequencies) } \\
(2 n=206)\end{array}$ \\
\hline Allele frequency of poly-T & & & \\
T5 & $57 / 178(32.02 \%)$ & $7 / 206(3.40 \%)$ & $<0.001$ \\
T7 & $117 / 178(65.73 \%)$ & $198 / 206(96.12 \%)$ & $<0.001$ \\
T9 & $4 / 178(2.25 \%)$ & $1 / 206(0.49 \%)$ & 0.286 \\
Allele frequency of TG-repeats & & $0 / 206$ & $0.464^{*}$ \\
TG10 & $1 / 178(0.56 \%)$ & $89 / 206(43.20 \%)$ & $<0.001$ \\
TG11 & $46 / 178(25.84 \%)$ & $115 / 206(55.83 \%)$ & 0.080 \\
TG12 & $115 / 178(64.61 \%)$ & $2 / 206(0.97 \%)$ & $<0.001$ \\
TG13 & $16 / 178(8.99 \%)$ & & \\
\hline
\end{tabular}

${ }^{*}$ Fisher's exact test.

TABLE 4: Frequencies of genotypes at poly-T polymorphic site.

\begin{tabular}{|c|c|c|c|c|c|}
\hline \multirow{2}{*}{ Type/number $(n)$} & \multicolumn{5}{|c|}{ Frequencies of genotypes at poly-T polymorphic site } \\
\hline & T5/T5 & $\mathrm{T} 5 / \mathrm{T} 7$ & T7/T7 & T5/T9 & T7/T9 \\
\hline CBAVD $(n=89)$ & $13 / 89(14.61 \%)$ & $28 / 89(31.46 \%)$ & $44 / 89(49.44 \%)$ & $3 / 89(3.37 \%)$ & $1 / 89(1.12 \%)$ \\
\hline Control $(n=103)$ & $0 / 103$ & $7 / 103(6.80 \%)$ & $95 / 103(92.23 \%)$ & $0 / 103$ & $1 / 103(0.97 \%)$ \\
\hline$P$ & $<0.001$ & $<0.001$ & $<0.001$ & 0.195 & $1.000^{*}$ \\
\hline
\end{tabular}

${ }^{*}$ Fisher's exact test.

performed with subjects from different regions of China. The study [16] reported that the frequency of 5T was about $44.5 \%$ in Chinese patients with congenital bilateral absence of the vas deferens and 13.46 in normal subjects, which were substantially higher than the findings reported [15]. Radpour et al. [3] found a 5T allele frequency of $25.94 \%$ among 106 CBAVD patients. In contrast, the authors did not detect the $5 \mathrm{~T}$ allele in a population of 43 normal male participants. The findings suggested that the distribution of poly-T polymorphism could vary in different regions, and more studies should be performed to further investigate this point. In our study, normal healthy Chinese people carry 7T or 9T most frequently. Shorter Tn tracts are associated with reduced levels of normal CFTR mRNA. CFTR mRNA splicing at exon 9 can be affected in 5T individuals, resulting in an mRNA subpopulation missing exon 9 that specifies a nonfunctional CFTR protein. The missing exon 9 in $5 \mathrm{~T}$ individuals often translates to CBAVD without other CF symptoms.

The 5T allele polymorphism varies considerably by geographic location, ranging from $0.0013 \%$ to $43.7 \%$ [18-22]; this polymorphism is particularly variable among non-Caucasian populations. We detected a T5 allele frequency of $32.02 \%$ among Chinese CBAVD patients, which is higher than that in the other report [3]. We also detected the T5 allele among normal Chinese males at a frequency of $3.40 \%$. The data is the most approximation to the previous report [8] suggesting that the T5 allele is insufficient to cause CBAVD. However, all the subjects carrying homozygous $5 \mathrm{~T}$ were patients with congenital bilateral absence of the vas deferens in the present study, while no subject with normal fertility was found to carry the homozygous 5T genotype, which further confirmed that shorter poly- $\mathrm{T}$ tracts are associated with more severe exon 9 splicing defects and a consequent functional decline in the CFTR protein. It is possible that the function of the CFTR protein is uniformly reduced among Chinese individuals compared with Caucasians.

Our results suggest that poly-T polymorphisms further affect the splicing of exon 9 when they precede TG-repeats. Compared with TG10 in a T7 background, Cuppens reported that the TG11 and TG12 alleles generated CFTR transcripts 
TABLE 5: Linkage of poly-T and TG-repeats.

\begin{tabular}{lccc}
\hline Linkage haplotypes & $\begin{array}{c}\text { CBAVD (frequencies) } \\
(2 n=178)\end{array}$ & $\begin{array}{c}\text { Control (frequencies) } \\
(2 n=206)\end{array}$ & $0.464^{*}$ \\
\hline TG10T9 & $1 / 178(0.56 \%)$ & $0 / 206$ & $<0.001$ \\
TG11T7 & $43 / 178(24.16 \%)$ & $88 / 206(42.72 \%)$ & 0.515 \\
TG11T9 & $3 / 178(1.69 \%)$ & $1 / 206(0.49 \%)$ & 0.034 \\
TG12T7 & $74 / 178(41.57 \%)$ & $108 / 206(52.43 \%)$ & $<0.001$ \\
TG12T5 & $41 / 178(23.03 \%)$ & $7 / 206(3.40 \%)$ & $0.501^{*}$ \\
TG13T7 & $0 / 178$ & $2 / 206(0.97 \%)$ & $<0.001$ \\
TG13T5 & $16 / 178(8.99 \%)$ & $0 / 206$ & \\
\hline
\end{tabular}

*Fisher's exact test.

TABLE 6: Linkage of poly-T, TG-repeat, and M470V.

\begin{tabular}{lccc}
\hline Linkage haplotypes & $\begin{array}{c}\text { CBAVD (frequencies) } \\
(2 n=88)\end{array}$ & $\begin{array}{c}\text { Control (frequencies) } \\
(2 n=114)\end{array}$ & 0.162 \\
\hline TG11T7-M470 & $3 / 88(3.41 \%)$ & $0 / 114$ & $<0.001$ \\
TG11T7-V470 & $23 / 88(26.14 \%)$ & $05 / 114(48.25 \%)$ & $0.189^{*}$ \\
TG11T9-M470 & $2 / 88(2.27 \%)$ & $1 / 114(0.88 \%)$ & $1.000^{*}$ \\
TG11T9-V470 & $1 / 88(1.14 \%)$ & $39 / 114(34.21 \%)$ & 0.596 \\
TG12T7-M470 & $27 / 88(30.68 \%)$ & $15 / 114(13.16 \%)$ & 0.239 \\
TG12T7-V470 & $7 / 88(7.95 \%)$ & $0 / 114$ & $0.436^{*}$ \\
TG12T5-M470 & $1 / 88(1.14 \%)$ & $3 / 114(2.63 \%)$ & $<0.001$ \\
TG12T5-V470 & $15 / 88(17.05 \%)$ & $1 / 114(0.88 \%)$ & $1.000^{*}$ \\
TG13T7-M470 & $0 / 88$ & $0 / 114$ & 0.002 \\
TG13T5-M470 & $9 / 88(10.23 \%)$ & $0 / 114$ & - \\
TG13T5-V470 & $0 / 88$ & & \\
\hline
\end{tabular}

${ }^{*}$ Fisher's exact test.

missing exon 9 at higher rates of 2.8- and 6-fold, respectively [23]. TG-repeats determine CFTR protein function and work in synergy with poly-T [24]. Longer TG-repeat tracts are associated with shorter poly-T tracts and elevated probabilities of exon 9 skipping [6]. Exon 9 encodes a portion of the CFTR protein's first nucleotide binding domain [25], and transcripts lacking exon 9 specify misfolded and dysfunctional CFTR chloride channels [26]. Fluid secretion is necessary for the normal development of renal tubules. If fluid secretion is blocked during embryonic development, renal tubular dysgenesis and early degradation occur [11, 27]. Because the vas deferens is highly sensitive to abnormal CFTR protein function, an increased risk of CBAVD maybe results from exon 9-null transcripts [28].

Fourteen of the CBAVD patients in our study were TG13T5 heterozygotes, supporting the theory that TGrepeats work with poly-T synergistically to precipitate CBAVD. We identified one TG13T5 homozygous CBAVD patient descended from a consanguineous union. To assess whether this genotype resulted from a genetic mutation, we recommended genetic testing of the patient's parents. In subsequent studies, we intend to expand our sample size to investigate the incidence of TG13T5. Our results confirm the importance of a follow-up study for the offspring of CBAVD patients who were conceived by intracytoplasmic sperm injection (ICSI) assisted reproductive treatment, to determine whether offspring carrying TG13T5 manifest as CBAVD. Five CBAVD patients in our study were TG12T5 homozygotes, whereas this genotype was not detected among the normal male participants. This finding confirms that TG-repeats function synergistically with poly-T to produce CBAVD.

Although no association between M470V and congenital bilateral absence of the vas deferens has been reported, M470V also may interact synergistically with poly-T and TGrepeats [29]. Our results show that the M470V allele alone is insufficient to produce CBAVD, but this polymorphism may contribute to CBAVD risk in individuals with short poly-T and long TG-repeat tracts, which is in agreement with the findings reported by Tomaiuolo et al. [30]. TG12T5-V470 haploid individuals are at greater risk of CBAVD. In our entire study population, no individuals carried the TG13T5-V470 haploid genotype; it is possible that this haploid genotype may cause a more severe phenotype. Therefore, examinations which could identify the genotypes of poly-T, TG repeats, and M470V of the CFTR gene in Chinese patients with CBAVD should and could be performed to avoid the inheritance of this disease to their children, thanks to the development of in vitro fertilization and ICSI technology [31]. Additional studies of larger populations are needed to address this question. 


\section{Conflicts of Interests}

The authors declare that they have no conflict of interests.

\section{Acknowledgment}

The Online Mendelian Inheritance of Man database (http://www.ncbi.nlm.nih.gov/omim/) was used to obtain data for this study (CBAVD [OMIM\#277180], CFTR [OMIM*602421], and CF [OMIM\#219700]).

\section{References}

[1] L. I. Lipshultz, S. S. Howards, and C. S. Niederberger, Infertility in the Male, Cambridge University Press, Cambridge, UK, 4th edition, 2009.

[2] J. Yu, Z. Chen, Y. Ni, and Z. Li, "CFTR mutations in men with congenital bilateral absence of the vas deferens (CBAVD): a systemic review and meta-analysis," Human Reproduction, vol. 27, no. 1, pp. 25-35, 2012.

[3] R. Radpour, M. A. Gilani, H. Gourabi, A. V. Dizaj, and S. Mollamohamadi, "Molecular analysis of the IVS8-T splice variant $5 \mathrm{~T}$ and M470V exon 10 missense polymorphism in Iranian males with congenital bilateral absence of the vas deferens," Molecular Human Reproduction, vol. 12, no. 7, pp. 469-473, 2006.

[4] A. de Meeus, C. Guittard, M. Desgeorges, S. Carles, J. Demaille, and M. Claustres, "Linkage disequilibrium between the M470V variant and the IVS8 polyT alleles of the CFTR gene in CBAVD," Journal of Medical Genetics, vol. 35, no. 7, pp. 594-596, 1998.

[5] E. Lopez, V. Viart, C. Guittard et al., "Variants in CFTR untranslated regions are associated with congenital bilateral absence of the vas deferens," Journal of Medical Genetics, vol. 48, no. 3, pp. 152-159, 2011.

[6] M. Claustres, "Molecular pathology of the CFTR locus in male infertility," Reproductive BioMedicine Online, vol. 10, no. 1, pp. 14-41, 2005.

[7] E. Zuccato, E. Buratti, C. Stuani, F. E. Baralle, and F. Pagani, "An intronic polypyrimidine-rich element downstream of the donor site modulates cystic fibrosis transmembrane conductance regulator exon 9 alternative splicing," The Journal of Biological Chemistry, vol. 279, no. 17, pp. 16980-16988, 2004.

[8] Q. Huang, W. Ding, and M. X. Wei, "Comparative analysis of common CFTR polymorphisms poly-T, TG-repeats and M470V in a healthy Chinese population," World Journal of Gastroenterology, vol. 14, no. 12, pp. 1925-1930, 2008.

[9] Y. Suzuki, R. Saito, H. Zaraket, C. Dapat, I. Caperig-Dapat, and H. Suzuki, "Rapid and specific detection of amantadineresistant influenza A viruses with a Ser31Asn mutation by the cycling probe method," Journal of Clinical Microbiology, vol. 48, no. 1, pp. 57-63, 2010.

[10] J. R. Riordan, J. M. Rommens, B. S. Kerem et al., "Identification of the cystic fibrosis gene: cloning and characterization of complementary DNA," Science, vol. 245, no. 4922, pp. 10661073, 1989.

[11] http://ghr.nlm.nih.gov/gene=CFTR.

[12] M. Ferrari and L. Cremonesi, "Genotype-phenotype correlation in cystic fibrosis patients," Annales de Biologie Clinique, vol. 54, no. 6, pp. 235-241, 1996.

[13] E. Dequeker, M. Stuhrmann, M. A. Morris et al., "Best practice guidelines for molecular genetic diagnosis of cystic fibrosis and CFTR-related disorders-updated European recommendations," European Journal of Human Genetics, vol. 17, no. 1, pp. 51-65, 2009.

[14] H. Li, Q. Wen, H. Li et al., "Mutations in the cystic fibrosis transmembrane conductance regulator (CFTR) in Chinese patients with congenital bilateral absence of vas deferens," Journal of Cystic Fibrosis, vol. 11, no. 4, pp. 316-323, 2012.

[15] H. S. Chiang, J. F. Lu, C. H. Liu, Y. N. Wu, and C. C. Wu, "CFTR (TG)m(T)n polymorphism in patients with CBAVD in a population expressing low incidence of cystic fibrosis," Clinical Genetics, vol. 76, no. 3, pp. 282-286, 2009.

[16] W. H. Ni, L. Jiang, Q. J. Fei et al., "The CFTR polymorphisms poly-T, TG-repeats and M470V in Chinese males with congenital bilateral absence of the vas deferens," Asian Journal of Andrology, vol. 14, no. 5, pp. 687-690, 2012.

[17] C. C. Wu, H. M. Hsieh-Li, Y. M. Lin, and H. S. Chiang, "Cystic fibrosis transmembrane conductance regulator gene screening and clinical correlation in Taiwanese males with congenital bilateral absence of the vas deferens," Human Reproduction, vol. 19, no. 2, pp. 250-253, 2004.

[18] T. Dörk, B. Dworniczak, C. Aulehla-Scholz et al., "Distinct spectrum of CFTR gene mutations in congenital absence of vas deferens," Human Genetics, vol. 100, no. 3-4, pp. 365-377, 1997.

[19] S. Leonardi, V. Bombace, N. Rotolo, C. Sciuto, and M. la Rosa, "Congenital absence of vas deferens and cystic fibrosis," Minerva Pediatrica, vol. 55, no. 1, pp. 43-50, 2003.

[20] W. Lissens, K. Z. Mahmoud, E. El-Gindi et al., "Molecular analysis of the cystic fibrosis gene reveals a high frequency of the intron 8 splice variant $5 \mathrm{~T}$ in Egyptian males with congenital bilateral absence of the vas deferens," Molecular Human Reproduction, vol. 5, no. 1, pp. 10-13, 1999.

[21] J. Zielenski, P. Patrizio, M. Corey et al., "CFTR gene variant for patients with congenital absence of vas deferens," The American Journal of Human Genetics, vol. 57, no. 4, pp. 958-960, 1995.

[22] C. Anzai, N. Morokawa, H. Okada, S. Kamidono, Y. Eto, and K. Yoshimura, "CFTR gene mutations in Japanese individuals with congenital bilateral absence of the vas deferens," Journal of Cystic Fibrosis, vol. 2, no. 1, pp. 14-18, 2003.

[23] M. H. Nam, M. Hijikata, L. A. Tuan et al., "Variations of the CFTR gene in the Hanoi-Vietnamese," The American Journal of Medical Genetics, vol. 136, no. 3, pp. 249-253, 2005.

[24] R. Radpour, H. Gourabi, A. V. Dizaj, W. Holzgreve, and X. Y. Zhong, "Genetic investigations of CFTR mutations in congenital absence of vas deferens, uterus, and vagina as a cause of infertility," Journal of Andrology, vol. 29, no. 5, pp. 506-513, 2008.

[25] C.-S. Chu, B. C. Trapnell, S. Curristin, G. R. Cutting, and R. G. Crystal, "Genetic basis of variable exon 9 skipping in cystic fibrosis transmembrane conductance regulator mRNA," Nature Genetics, vol. 3, no. 2, pp. 151-156, 1993.

[26] S. J. Delaney, D. P. Rich, S. A. Thomson et al., "Cystic fibrosis transmembrane conductance regulator splice variants are not conserved and fail to produce chloride channels," Nature Genetics, vol. 4, no. 4, pp. 426-430, 1993.

[27] I. Sermet-Gaudelus, M. Déchaux, B. Vallée et al., "Chloride transport in nasal ciliated cells of cystic fibrosis heterozygotes," The American Journal of Respiratory and Critical Care Medicine, vol. 171, no. 9, pp. 1026-1031, 2005.

[28] M. Chillon, T. Casals, B. Mercier et al., "Mutations in the cystic fibrosis gene in patients with congenital absence of the vas deferens," The New England Journal of Medicine, vol. 332, no. 22, pp. 1475-1480, 1995. 
[29] M. Boudaya, S. H. Fredj, R. B. Haj et al., "Cystic fibrosis transmembrane conductance regulator mutations and polymorphisms associated with congenital bilateral absence of vas deferens in a restricted group of patients from North Africa," Annals of Human Biology, vol. 39, no. 1, pp. 76-79, 2012.

[30] R. Tomaiuolo, M. Fausto, A. Elce et al., "Enhanced frequency of CFTR gene variants in couples who are candidates for assisted reproductive technology treatment," Clinical Chemistry and Laboratory Medicine, vol. 49, no. 8, pp. 1289-1293, 2011.

[31] E. Mocanu, R. Shattock, D. Barton et al., "All azoospermic males should be screened for cystic fibrosis mutations before intracytoplasmic sperm injection," Fertility and Sterility, vol. 94, no. 6, pp. 2448-2450, 2010. 


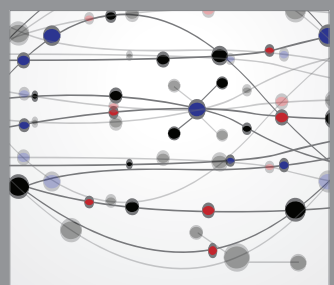

The Scientific World Journal
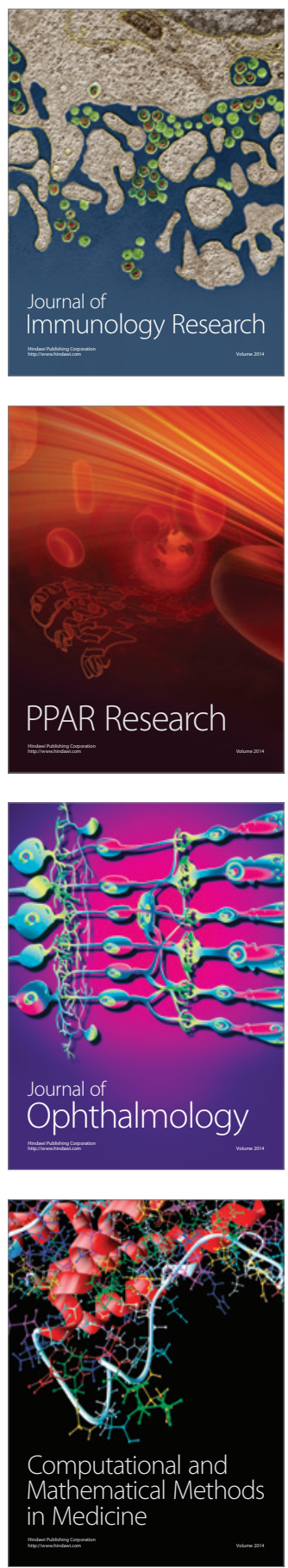

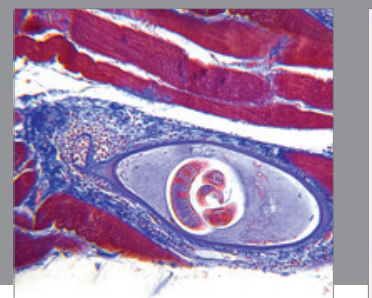

Gastroenterology

Research and Practice
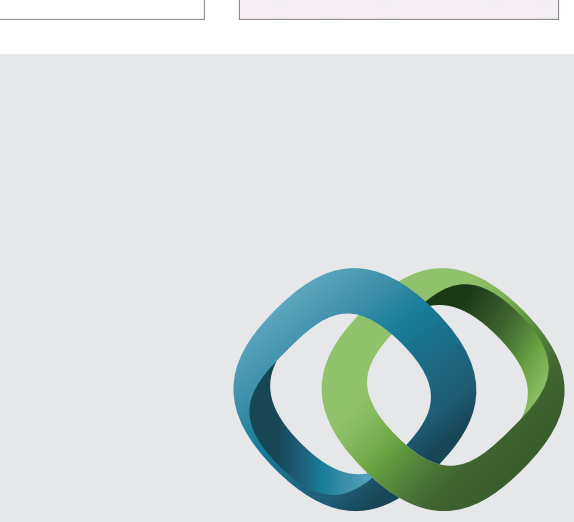

\section{Hindawi}

Submit your manuscripts at

http://www.hindawi.com
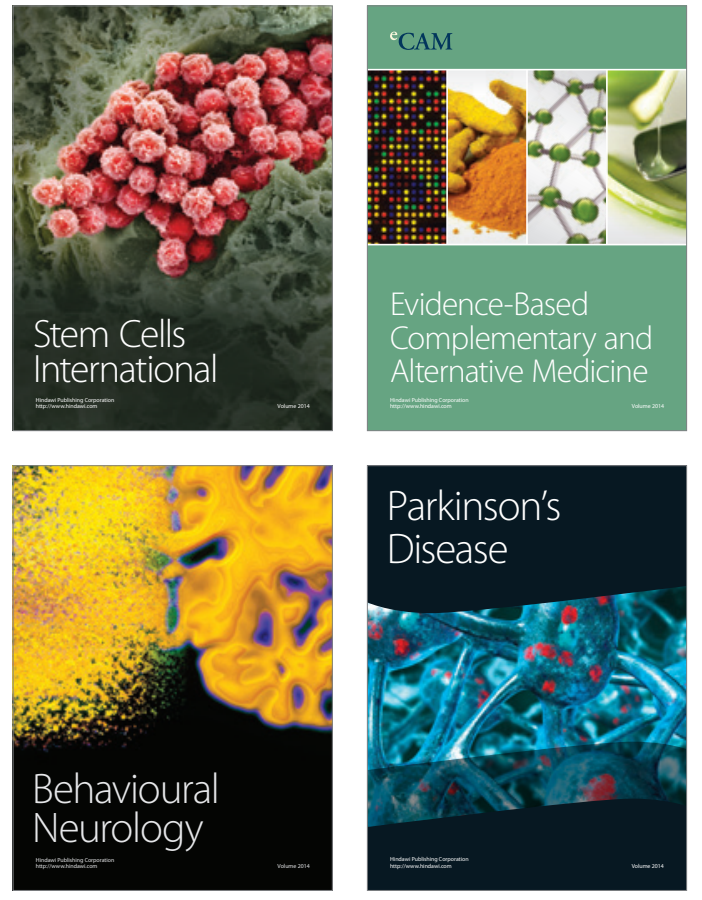
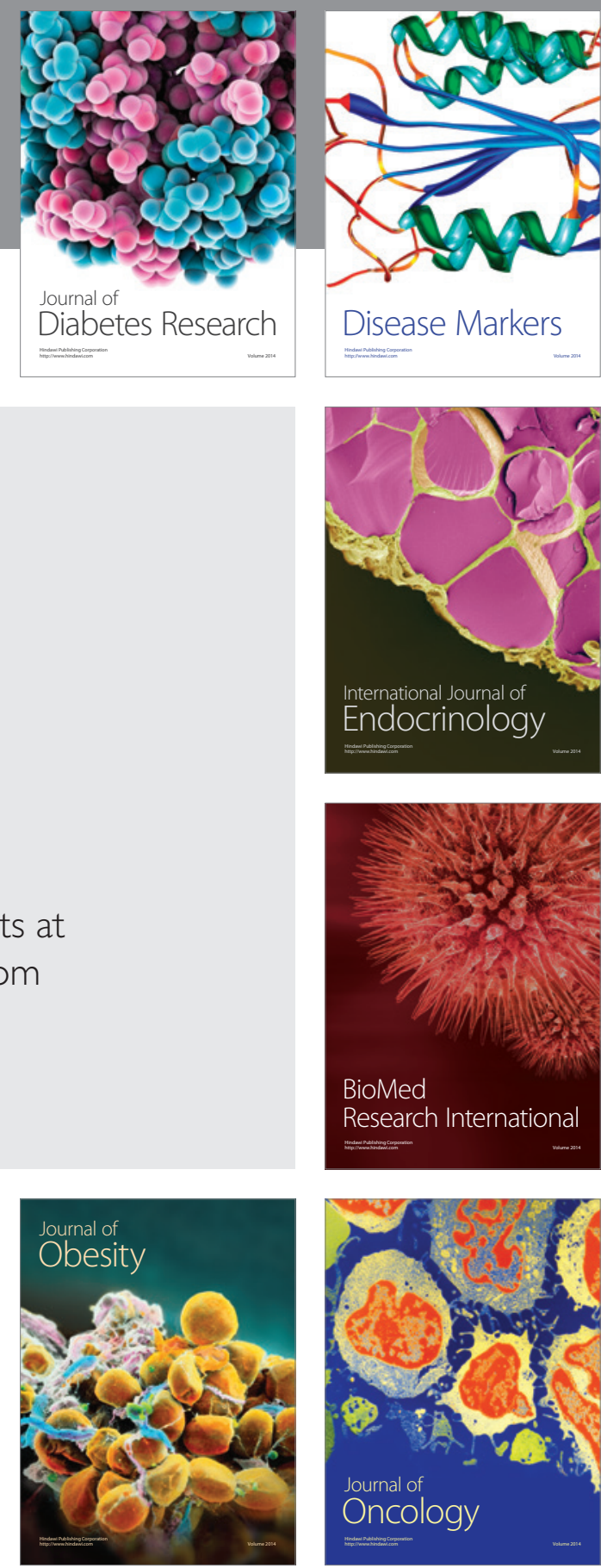

Disease Markers
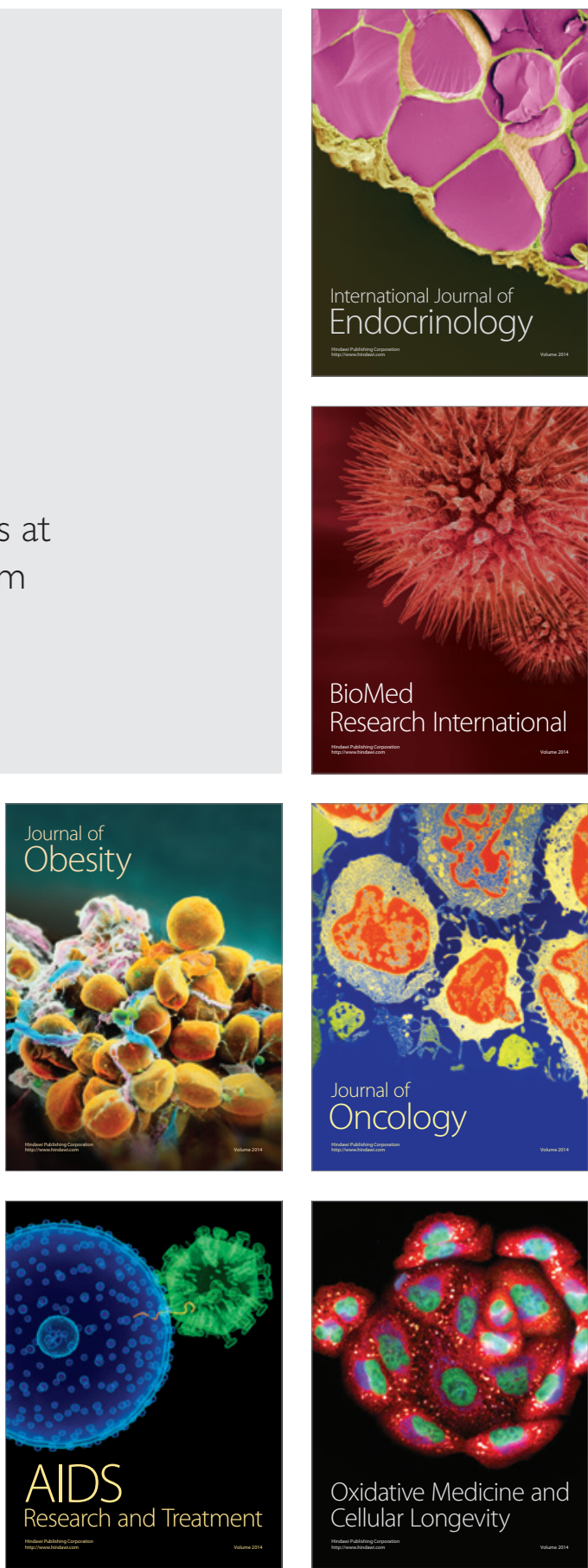